Bull. Korean Math. Soc. 50 (2013), No. 2, pp. 627-637

http://dx.doi.org/10.4134/BKMS.2013.50.2.627

\title{
MAXIMAL PROPERTIES OF SOME SUBSEMIBANDS OF ORDER-PRESERVING FULL TRANSFORMATIONS
}

\author{
Ping Zhao And Mei Yang
}

\begin{abstract}
Let $[n]=\{1,2, \ldots, n\}$ be ordered in the standard way. The order-preserving full transformation semigroup $\mathcal{O}_{n}$ is the set of all orderpreserving singular full transformations on $[n]$ under composition. For this semigroup we describe maximal subsemibands, maximal regular subsemibands, locally maximal regular subsemibands, and completely obtain their classification.
\end{abstract}

\section{Introduction}

A semigroup is called idempotent-generated or semiband if it is generated by its idempotents. The latter term was introduced by F. Pastijn [7].

Let $[n]=\{1,2, \ldots, n\}$ ordered in the standard way. We denote by $\mathcal{S i n g}_{n}$ the semigroup (under composition) of all singular full transformations on $[n]$. We say that a full transformation $\alpha$ in $\mathcal{S}_{\text {ing }}$ is order-preserving if, for all $x, y \in[n]$, $x \leq y$ implies $x \alpha \leq y \alpha$. We denote by $\mathcal{O}_{n}$ the subsemigroup of $\mathcal{S i n g}_{n}$ of all order-preserving singular full transformations.

The semigroup $\mathcal{O}_{n}$ was studied first by Aizenstat [1] and subsequently by many authors (see, for example [2-6], [8-13]). In particular, Howie [5] proved that $\mathcal{O}_{n}$ is a regular semiband. Garba [2] further proved that each one of the ideals of $\mathcal{O}_{n}$ is also a regular semiband. Yang [12] classified completely maximal subsemibands and maximal regular subsemibands of $\mathcal{O}_{n}$. Recently, Xu, Zhao and $\mathrm{Li}[9]$ obtained a complete classification of locally maximal subsemibands of $\mathcal{O}_{n}$. Further, Zhao, Xu and Yang [13] obtained a simpler form of the classification of maximal (regular) subsemibands of $\mathcal{O}_{n}$, using results of Xu, Zhao and $\operatorname{Li}[9]$.

In view of the above work, it is natural to seek a description of the locally maximal regular subsemibands of $\mathcal{O}_{n}$. In Section 2 we obtain a same simpler form of the classification of maximal (regular) subsemibands of $\mathcal{O}_{n}$, using a

Received December 12, 2011; Revised May 11, 2012.

2010 Mathematics Subject Classification. 20M20.

Key words and phrases. order-preserving full transformation semigroup, maximal subsemiband, maximal regular subsemiband, locally maximal subsemiband, locally maximal regular subsemiband.

This work is supported by Natural Science Fund of Guizhou(No. [2010] 3174). 
different approach from Zhao, $\mathrm{Xu}$ and Yang [13]. In Section 3 we obtain a classification of locally maximal regular subsemibands of $\mathcal{O}_{n}$.

From Gomes and Howie [3], Green's equivalences on $\mathcal{O}_{n}$ are characterized as:

$$
\begin{aligned}
\alpha \mathcal{L} \beta & \Leftrightarrow i m(\alpha)=i m(\beta), \\
\alpha \mathcal{R} \beta & \Leftrightarrow \operatorname{ker}(\alpha)=\operatorname{ker}(\beta), \\
\alpha \mathcal{J} \beta & \Leftrightarrow|\operatorname{im}(\alpha)|=|i m(\beta)| .
\end{aligned}
$$

Thus $\mathcal{O}_{n}$ has $n-1 \mathcal{J}$-classes: $J_{1}, J_{2}, \ldots, J_{n-1}$, where $J_{r}=\left\{\alpha \in \mathcal{O}_{n}:|i m(\alpha)|=\right.$ $r\}$.

Gomes and Howie [3] used the notation $[i \rightarrow i+1]$ for the increasing idempotent $e$ defined by $i e=i+1, x e=x(x \neq i)$, and $[i \rightarrow i-1]$ for the decreasing idempotent $f$ defined by if $=i-1, x f=x(x \neq i)$. As usual, we denote by $E(S)$ the set of all idempotents of a subset $S$ of $\mathcal{O}_{n}$. Let $E_{n-1}^{+}=\{[i \rightarrow i+1]$ : $1 \leq i \leq n-1\}$ and $E_{n-1}^{-}=\{[i \rightarrow i-1]: 2 \leq i \leq n\}$ be the increasing and decreasing idempotent sets, respectively. Then $E\left(J_{n-1}\right)=E_{n-1}^{+} \cup E_{n-1}^{-}$.

\section{Maximal (regular) subsemibands of $\mathcal{O}_{n}$}

Both maximal subsemibands and maximal regular subsemibands of $\mathcal{O}_{n}$ were studied by [12]. Zhao, Xu and Yang [13] obtained a simpler form of the classification of maximal (regular) subsemibands of $\mathcal{O}_{n}$, using results of Xu, Zhao and $\mathrm{Li}[9]$. In this section, we obtain a same simpler form of the classification of maximal (regular) subsemibands of $\mathcal{O}_{n}$, using a different approach from Zhao, Xu and Yang [13]. For convenience, we introduce the following notation from $[8]$.

Let

$$
\begin{aligned}
& \mathcal{C}_{n}^{-}=\left\{\alpha \in \mathcal{O}_{n}:(\forall x \in[n]) x \alpha \leq x\right\}, \\
& \mathcal{C}_{n}^{+}=\left\{\alpha \in \mathcal{O}_{n}:(\forall x \in[n]) x \alpha \geq x\right\},
\end{aligned}
$$

be the semigroups of all singular order-preserving and decreasing full transformations and order-preserving and increasing full transformations on $[n]$, respectively.

As in [4], for any $\alpha \in \mathcal{O}_{n}$, let

$$
\begin{aligned}
& x \alpha^{-}= \begin{cases}x \alpha, & x \in[n]_{\alpha}^{-} \\
x, & \text { otherwise }\end{cases} \\
& x \alpha^{+}= \begin{cases}x \alpha, & x \in[n]_{\alpha}^{+} \\
x, & \text { otherwise }\end{cases}
\end{aligned}
$$

where $[n]_{\alpha}^{-}=\{x \in[n]: x \alpha \leq x\}$, and $[n]_{\alpha}^{+}=\{x \in[n]: x \alpha \geq x\}$. It is obvious that $\alpha^{-} \in \mathcal{C}_{n}^{-}$and $\alpha^{+} \in \mathcal{C}_{n}^{+}$. The following lemma was proved by Higgins [4, page 1053]. 
Lemma 2.1. Let $\alpha \in \mathcal{O}_{n}$. Then

$$
\alpha=\alpha^{+} \alpha^{-}=\alpha^{-} \alpha^{+},
$$

with $\alpha^{-} \in \mathcal{C}_{n}^{-}, \alpha^{+} \in \mathcal{C}_{n}^{+}$.

For convenience, we use $[n \rightarrow n+1]$ or $[1 \rightarrow 0]$ to denote $\emptyset$ (the empty mapping). With this notation, we have:

Lemma 2.2. Let $\alpha \in \mathcal{C}_{n}^{+}$. If $k \alpha=k$ for some $1 \leq k \leq n$, then

$$
\alpha \in\left\langle E_{n-1}^{+} \backslash\{[k \rightarrow k+1]\}\right\rangle .
$$

Proof. Since $\alpha \in \mathcal{C}_{n}^{+} \subseteq \mathcal{O}_{n}$, we have that the $\operatorname{ker}(\alpha)$-classes are convex subsets $C$ of $[n]$, in the sense that

$$
x, y \in C \text { and } x \leq z \leq y \Longrightarrow z \in C .
$$

Then $\alpha$ can be expressed as

$$
\alpha=\left(\begin{array}{llll}
A_{1} & A_{2} & \cdots & A_{r} \\
b_{1} & b_{2} & \cdots & b_{r}
\end{array}\right),
$$

where $A_{i}=\left\{a_{i}, a_{i}+1, \ldots, a_{i+1}-1\right\}(1 \leq i \leq r-1), A_{r}=\left\{a_{r}, a_{r}+1, \ldots, n\right\}$, $1=a_{1}<a_{2}<\cdots<a_{r}$ and $b_{1}<b_{2}<\cdots<b_{r}$. Since $\alpha \in \mathcal{C}_{n}^{+}$, we have

$$
\begin{aligned}
& a_{i}=\min A_{i} \leq \max A_{i} \leq\left(\max A_{i}\right) \alpha=b_{i}, 1 \leq i \leq r-1, \\
& a_{r}=\min A_{r} \leq \max A_{r}(=n) \leq\left(\max A_{r}\right) \alpha=b_{r} .
\end{aligned}
$$

Thus

$$
b_{r}=n \text { and } a_{i} \leq b_{i}, i \in[n] .
$$

Let $e_{0}$ be the identity mapping on $[n]$, and let

$$
\begin{gathered}
E^{+}(i, j)=[i \rightarrow i+1] \cdot[i+1 \rightarrow i+2] \cdots[j-1 \rightarrow j], 1 \leq i<j \leq n, \\
E^{+}(i, i)=e_{0}, i \in[n] .
\end{gathered}
$$

Further, let

$$
\beta=E^{+}\left(a_{r}, b_{r}\right) E^{+}\left(a_{r-1}, b_{r-1}\right) \cdots E^{+}\left(a_{1}, b_{1}\right) .
$$

We claim that $\alpha=\beta$. To prove that $\alpha=\beta$, take any $x \in[n]$. Suppose that $x \in A_{s}(1 \leq s \leq r)$. Then

$$
x \beta=x E^{+}\left(a_{r}, b_{r}\right) E^{+}\left(a_{r-1}, b_{r-1}\right) \cdots E^{+}\left(a_{1}, b_{1}\right)=b_{s}=x \alpha .
$$

Note that $[n \rightarrow n+1]=\emptyset$ (the empty mapping). If $k=n$, then $\alpha=\beta \in$ $\left\langle E_{n-1}^{+} \cup\left\{e_{0}\right\}\right\rangle=\left\langle E_{n-1}^{+} \backslash\{[n \rightarrow n+1]\}\right\rangle \cup\left\{e_{0}\right\}$. Since $\alpha \in \mathcal{C}_{n}^{+} \subseteq \mathcal{O}_{n} \subseteq$ Sing $_{n}$, we have $\alpha \in\left\langle E_{n-1}^{+} \backslash\{[n \rightarrow n+1]\}\right\rangle$. If $1 \leq k \leq n-1$. Note that $b_{r}=n$. Since $k \alpha=k$, there exists $1 \leq j \leq r-1$ such that $k \in A_{j}$ and $b_{j}=k$. Since $\alpha \in \mathcal{C}_{n}^{+}$, we have $b_{j}=\max A_{j}=k$ and so $a_{j+1}=\min A_{j+1}=k+1$. Thus

(2.1) $[k \rightarrow k+1] \notin\left\{\left[a_{i} \rightarrow a_{i}+1\right],\left[a_{i}+1 \rightarrow a_{i}+2\right], \ldots,\left[b_{i}-1 \rightarrow b_{i}\right]\right\}, a_{i}<b_{i}$.

Note that $E^{+}\left(a_{i}, b_{i}\right)=\left[a_{i} \rightarrow a_{i}+1\right]\left[a_{i}+1 \rightarrow a_{i}+2\right] \cdots\left[b_{i}-1 \rightarrow b_{i}\right]$ if $a_{i}<b_{i}$; $E^{+}\left(a_{i}, b_{i}\right)=e_{0}$ if $a_{i}=b_{i}$. It follows immediately from (2.1) that

$$
E^{+}\left(a_{i}, b_{i}\right) \in\left\langle E_{n-1}^{+} \backslash\{[k \rightarrow k+1]\} \cup\left\{e_{0}\right\}\right\rangle, 1 \leq i \leq r .
$$


Then $\alpha=\beta \in\left\langle E_{n-1}^{+} \backslash\{[k \rightarrow k+1]\} \cup\left\{e_{0}\right\}\right\rangle$. It is obvious that $\left\langle E_{n-1}^{+} \backslash\{[k \rightarrow\right.$ $\left.k+1]\} \cup\left\{e_{0}\right\}\right\rangle=\left\langle E_{n-1}^{+} \backslash\{[k \rightarrow k+1]\}\right\rangle \cup\left\{e_{0}\right\}$. Since $\alpha \in \mathcal{C}_{n}^{+} \subseteq \mathcal{O}_{n} \subseteq \mathcal{S}_{\text {ing }}$, we have

$$
\alpha \in\left\langle E_{n-1}^{+} \backslash\{[k \rightarrow k+1]\}\right\rangle .
$$

Similarly, we can prove:

Lemma 2.3. Let $\alpha \in \mathcal{C}_{n}^{-}$. If $k \alpha=k$ for some $1 \leq k \leq n$. Then

$$
\alpha \in\left\langle E_{n-1}^{-} \backslash\{[k \rightarrow k-1]\}\right\rangle \text {. }
$$

The following lemma is immediate by definition of $\alpha^{+}, \alpha^{-}$:

Lemma 2.4. For any $\alpha \in \mathcal{O}_{n}$, we have

(i) If $k \alpha \leq k$ for some $1 \leq k \leq n$, then $k \alpha^{+}=k$.

(ii) If $k \alpha \geq k$ for some $1 \leq k \leq n$, then $k \alpha^{-}=k$.

For any $s, t \in[n]$, let

$$
M_{s t}=\left\{\alpha \in \mathcal{O}_{n}: s \alpha \leq s, t \alpha \geq t\right\} .
$$

With above notation, we have:

Lemma 2.5. Let $n \geq 3$. Then

$$
M_{s t}=\left\langle E\left(J_{n-1}\right) \backslash\{[s \rightarrow s+1],[t \rightarrow t-1]\}\right\rangle, s, t \in[n] .
$$

Proof. Let $P_{s t}=\left\langle E\left(J_{n-1}\right) \backslash\{[s \rightarrow s+1],[t \rightarrow t-1]\}\right\rangle$. It is easy to prove that $M_{s t}$ is a subsemigroup of $\mathcal{O}_{n}$. It is obvious that $E\left(J_{n-1}\right) \backslash\{[s \rightarrow s+1],[t \rightarrow$ $t-1]\} \subseteq M_{s t}$. Then $P_{s t}=\left\langle E\left(J_{n-1}\right) \backslash\{[s \rightarrow s+1],[t \rightarrow t-1]\}\right\rangle \subseteq M_{s t}$.

It remains to prove that $M_{s t} \subseteq P_{s t}$. Let $\alpha \in M_{s t} \subseteq \mathcal{O}_{n}$. By Lemmas 2.1 and 2.4, we have

$$
\alpha=\alpha^{+} \alpha^{-}=\alpha^{-} \alpha^{+}, \quad \alpha^{-} \in \mathcal{C}_{n}^{-}, \quad \alpha^{+} \in \mathcal{C}_{n}^{+},
$$

and $s \alpha^{+}=s, t \alpha^{-}=t$. Note that $E\left(J_{n-1}\right)=E_{n-1}^{+} \cup E_{n-1}^{-}$. Thus, by Lemmas 2.2 and 2.3 ,

$$
\begin{aligned}
\alpha & =\alpha^{+} \alpha^{-} \in\left\langle E_{n-1}^{+} \backslash\{[s \rightarrow s+1]\}\right\rangle \cdot\left\langle E_{n-1}^{-} \backslash\{[t \rightarrow t-1]\}\right\rangle \\
& \subseteq\left\langle E\left(J_{n-1}\right) \backslash\{[s \rightarrow s+1],[t \rightarrow t-1]\}\right\rangle=P_{s t} .
\end{aligned}
$$

A subsemiband $S$ of $\mathcal{O}_{n}$ is called maximal subsemiband if for an arbitrary subsemiband $T$ of $\mathcal{O}_{n}$ such that $S \subset T$, then $T=\mathcal{O}_{n}$. Combining [12, Theorem 2.1 and Lemma 2.3], we obtain the following.

Lemma 2.6. Let $n \geq 3$. Let $I_{n-2}=\left\{\alpha \in \mathcal{O}_{n}:|i m(\alpha)| \leq n-2\right\}$. Then each maximal subsemiband of $\mathcal{O}_{n}$ must be one of the following forms:

(C) $C_{s}=I_{n-2} \cup\left\langle E\left(J_{n-1}\right) \backslash\{[s \rightarrow s+1]\rangle, s=1,2, \ldots, n-1\right.$.

(D) $D_{s}=I_{n-2} \cup\left\langle E\left(J_{n-1}\right) \backslash\{[s \rightarrow s-1]\rangle, s=2,3, \ldots, n\right.$.

Now, it is easy to prove one of the main results of this section: 
Theorem 2.7. Let $n \geq 3$. Let $I_{n-2}=\left\{\alpha \in \mathcal{O}_{n}:|i m(\alpha)| \leq n-2\right\}$. Then each maximal subsemiband of $\mathcal{O}_{n}$ must be one of the following forms:

(A) $A_{s}=I_{n-2} \cup\left\{\alpha \in \mathcal{O}_{n}: s \alpha \leq s\right\}, s=1,2, \ldots, n-1$.

(B) $B_{s}=I_{n-2} \cup\left\{\alpha \in \mathcal{O}_{n}: s \alpha \geq s\right\}, s=2,3, \ldots, n$.

Proof. Let $M_{s t}$ be as defined in (2.2). Then $M_{s 1}=\left\{\alpha \in \mathcal{O}_{n}: s \alpha \leq s\right\}$ and $M_{n s}=\left\{\alpha \in \mathcal{O}_{n}: s \alpha \geq s\right\}$. Note that $[1 \rightarrow 0]=[n \rightarrow n+1]=\emptyset$ (the empty mapping). Thus, by Lemma 2.5,

$$
\begin{aligned}
A_{s} & =I_{n-2} \cup\left\{\alpha \in \mathcal{O}_{n}: s \alpha \leq s\right\}=I_{n-2} \cup M_{s 1} \\
& =I_{n-2} \cup\left\langle E\left(J_{n-1}\right) \backslash\{[s \rightarrow s+1]\rangle=C_{s},\right. \\
B_{s} & =I_{n-2} \cup\left\{\alpha \in \mathcal{O}_{n}: s \alpha \geq s\right\}=I_{n-2} \cup M_{n s} \\
& =I_{n-2} \cup\left\langle E\left(J_{n-1}\right) \backslash\{[s \rightarrow s-1]\rangle=D_{s} .\right.
\end{aligned}
$$

Hence Theorem 2.7 holds by Lemma 2.6.

A regular subsemiband $S$ of $\mathcal{O}_{n}$ is called maximal regular subsemiband if for an arbitrary regular subsemiband $T$ of $\mathcal{O}_{n}$ such that $S \subset T$, then $T=\mathcal{O}_{n}$. Note that $[1 \rightarrow 0]=[n \rightarrow n+1]=\emptyset$ (the empty mapping). Combining [12, Lemma 2.3 and Theorem 4.1], we obtain the following.

Lemma 2.8. Let $n \geq 4$. Let $I_{n-2}=\left\{\alpha \in \mathcal{O}_{n}:|i m(\alpha)| \leq n-2\right\}$. Then each maximal regular subsemiband of $\mathcal{O}_{n}$ must be the following forms:

(F) $F_{s}=I_{n-2} \cup\left\langle E\left(J_{n-1}\right) \backslash\{[s \rightarrow s+1],[s \rightarrow s-1]\}\right\rangle, s=1,2, \ldots, n$.

(G) $G_{s}=I_{n-2} \cup\left\langle E\left(J_{n-1}\right) \backslash\{[s \rightarrow s+1],[s+1 \rightarrow s]\}\right\rangle, s=2,3, \ldots, n-2$.

Using Lemma 2.5 and Lemma 2.8, the other main result of this section is now established:

Theorem 2.9. Let $n \geq 4$. Let $I_{n-2}=\left\{\alpha \in \mathcal{O}_{n}:|i m(\alpha)| \leq n-2\right\}$. Then each maximal regular subsemiband of $\mathcal{O}_{n}$ must be the following forms:

(A) $A_{s}=I_{n-2} \cup\left\{\alpha \in \mathcal{O}_{n}: s \alpha=s\right\}, s=1,2, \ldots, n$.

(B) $B_{s}=I_{n-2} \cup\left\{\alpha \in \mathcal{O}_{n}: s \alpha \leq s,(s+1) \alpha \geq s+1\right\}, s=2,3, \ldots, n-2$.

Proof. Let $M_{s t}$ be as defined in (2.2). Then $M_{s s}=\left\{\alpha \in \mathcal{O}_{n}: s \alpha=s\right\}$ and $M_{s(s+1)}=\left\{\alpha \in \mathcal{O}_{n}: s \alpha \leq s,(s+1) \alpha \geq s+1\right\}$. Note that $[1 \rightarrow 0]=[n \rightarrow$ $n+1]=\emptyset$ (the empty mapping). Thus, by Lemma 2.5,

$$
\begin{gathered}
A_{s}=I_{n-2} \cup\left\{\alpha \in \mathcal{O}_{n}: s \alpha=s\right\}=I_{n-2} \cup M_{s s} \\
=I_{n-2} \cup\left\langle E\left(J_{n-1}\right) \backslash\{[s \rightarrow s+1],[s \rightarrow s-1]\}\right\rangle=F_{s}, \\
B_{s}=I_{n-2} \cup\left\{\alpha \in \mathcal{O}_{n}: s \alpha \leq s,(s+1) \alpha \geq s+1\right\}=I_{n-2} \cup M_{s(s+1)} \\
=I_{n-2} \cup\left\langle E\left(J_{n-1}\right) \backslash\{[s \rightarrow s+1],[s+1 \rightarrow s]\}\right\rangle=G_{s} .
\end{gathered}
$$

Hence Theorem 2.9 holds by Lemma 2.8 . 


\section{Locally maximal regular subsemibands of $\mathcal{O}_{n}$}

Let $I$ be a subset of $E\left(J_{n-1}\right)$. A subsemiband $\langle I\rangle$ of $\mathcal{O}_{n}$ is called a locally maximal regular subsemiband if $\langle I\rangle$ is regular, and any regular subsemiband $\langle J\rangle\left(J \subseteq E\left(J_{n-1}\right)\right)$ of $\mathcal{O}_{n}$ properly containing $\langle I\rangle$ must be $\mathcal{O}_{n}$. In this section, we obtain a classification of locally maximal regular subsemibands of $\mathcal{O}_{n}$.

The main result of this section is:

Theorem 3.1. Let $n \geq 4$. Let $I_{n-2}=\left\{\alpha \in \mathcal{O}_{n}:|i m(\alpha)| \leq n-2\right\}$. Then each locally maximal regular subsemiband of $\mathcal{O}_{n}$ must be the following forms:

(A) $A_{s}=\left\{\alpha \in \mathcal{O}_{n}: s \alpha=s\right\}, s=1,2, \ldots, n$.

(B) $B_{s}=\left\{\alpha \in \mathcal{O}_{n}: s \alpha \leq s,(s+1) \alpha \geq s+1\right\}, s=2,3, \ldots, n-2$.

To prove Theorem 3.1, we begin by establishing a series of lemmas. Combining [5, Lemmas 1.2 and 1.3], we know that $\mathcal{O}_{n}$ is generated by $E\left(J_{n-1}\right)$. Note that $\left|E\left(J_{n-1}\right)\right|=2 n-2$. From the result [3, Theorem 2.8] that the rank of $\mathcal{O}_{n}$ is $2 n-2$, we immediately deduce:

Lemma 3.2. Let $n \geq 4$. Then

$$
\mathcal{O}_{n}=\left\langle E\left(J_{n-1}\right)\right\rangle \text { and no proper subset of } E\left(J_{n-1}\right) \text { can generate } \mathcal{O}_{n} \text {. }
$$

It is well known that the characterized forms of the Green's relations in $\mathcal{S i n g}_{n}$ are the same as in $\mathcal{O}_{n}$ (see Section 1). $\mathcal{S}_{\text {ing }}$ has $n-1 \mathcal{J}$-classes: $S J_{r}=\left\{\alpha \in \operatorname{Sing}_{n}:|i m(\alpha)|=r\right\}, r=1,2, \ldots, n-1$. Let

$$
S I_{r}=\left\{\alpha \in \operatorname{Sing}_{n}:|i m(\alpha)| \leq r\right\}, r=1,2, \ldots, n-1 .
$$

Then the sets $S I_{r}$ are two-sided ideal of $\mathcal{S}_{i n g}$. As usual, we denote by $E(S)$ the set of all idempotents of a subset $S$ of $\mathcal{S i n g}_{n}$. Let $I$ be nonempty subsets of $E\left(S J_{n-1}\right)$. It is obvious that $I \subseteq E\left(\langle I\rangle \cap S J_{n-1}\right)$. In general, $E\left(\langle I\rangle \cap S J_{n-1}\right) \subseteq$ $I$ is false. For example, let

$$
f=\left(\begin{array}{ccccc}
2 & 3 & \cdots & n-1 & \{n, 1\} \\
2 & 3 & \cdots & n-1 & 1
\end{array}\right), g=\left(\begin{array}{ccccc}
2 & 3 & \cdots & n-1 & \{n, 1\} \\
2 & 3 & \cdots & n-1 & n
\end{array}\right),
$$

then $f, g \in E\left(S J_{n-1}\right)$. Let $\eta=f \cdot[n-1 \rightarrow n] \cdot[n-2 \rightarrow n-1] \cdots[1 \rightarrow 2]$, then

$$
\eta=\left(\begin{array}{ccccc}
2 & 3 & \cdots & n-1 & \{n, 1\} \\
3 & 4 & \cdots & n & 2
\end{array}\right)
$$

and so $\eta^{n-1}=g$. Clearly, $E_{n-1}^{+} \subseteq E\left(S J_{n-1}\right)$. Let $I=E_{n-1}^{+} \cup\{f\}$. Then $g=$ $\eta^{n-1} \in\langle I\rangle$ and so $g \in E\left(\langle I\rangle \cap S J_{n-1}\right)$. Clearly, $g \notin I$. Thus $E\left(\langle I\rangle \cap S J_{n-1}\right) \nsubseteq I$. However, using Lemma 3.2, we have the following.

Lemma 3.3. Let $I$ be a subset of $E\left(J_{n-1}\right)$. Then

$$
E\left(\langle I\rangle \cap J_{n-1}\right)=I .
$$

Proof. Clearly, $I \subseteq E\left(\langle I\rangle \cap J_{n-1}\right)$. Now, we need to prove that $E\left(\langle I\rangle \cap J_{n-1}\right) \subseteq$ I. Note that $I \subseteq E\left(\langle I\rangle \cap J_{n-1}\right) \subseteq\langle I\rangle$. Then $\langle I\rangle \subseteq\left\langle E\left(\langle I\rangle \cap J_{n-1}\right)\right\rangle \subseteq\langle\langle I\rangle\rangle=\langle I\rangle$ 
and so $\left\langle E\left(\langle I\rangle \cap J_{n-1}\right)\right\rangle=\langle I\rangle$. Let $I^{*}=E\left(\langle I\rangle \cap J_{n-1}\right) \backslash I$. Then $I \subseteq E\left(J_{n-1}\right) \backslash I^{*}$ and so $\left\langle I^{*}\right\rangle \subseteq\left\langle E\left(\langle I\rangle \cap J_{n-1}\right)\right\rangle=\langle I\rangle \subseteq\left\langle E\left(J_{n-1}\right) \backslash I^{*}\right\rangle$. Thus

$$
E\left(J_{n-1}\right)=I^{*} \cup\left(E\left(J_{n-1}\right) \backslash I^{*}\right) \subseteq\left\langle I^{*}\right\rangle \cup\left\langle E\left(J_{n-1}\right) \backslash I^{*}\right\rangle=\left\langle E\left(J_{n-1}\right) \backslash I^{*}\right\rangle
$$

and so $\left\langle E\left(J_{n-1}\right)\right\rangle \subseteq\left\langle E\left(J_{n-1}\right) \backslash I^{*}\right\rangle \subseteq \mathcal{O}_{n}$. It follows immediately form Lemma 3.2 that

$$
E\left(J_{n-1}\right) \backslash I^{*}=E\left(J_{n-1}\right) .
$$

Then $I^{*}=\emptyset$ (the empty set) and so $E\left(\langle I\rangle \cap J_{n-1}\right) \subseteq I$.

Further, we have:

Lemma 3.4. Let $I_{n-2}=\left\{\alpha \in \mathcal{O}_{n}:|i m(\alpha)| \leq n-2\right\}$. Let $I$ and $J$ be nonempty subsets of $E\left(J_{n-1}\right)$. Then

(i) $I \subseteq J \Leftrightarrow\langle I\rangle \subseteq\langle J\rangle \Leftrightarrow I_{n-2} \cup\langle I\rangle \subseteq I_{n-2} \cup\langle J\rangle$.

(ii) $I \subset J \Leftrightarrow\langle I\rangle \subset\langle J\rangle \Leftrightarrow I_{n-2} \cup\langle I\rangle \subset I_{n-2} \cup\langle J\rangle$.

Proof. (i) Clearly,

$$
I \subseteq J \Rightarrow\langle I\rangle \subseteq\langle J\rangle \Rightarrow I_{n-2} \cup\langle I\rangle \subseteq I_{n-2} \cup\langle J\rangle .
$$

To prove that

$$
I \subseteq J \Leftarrow\langle I\rangle \subseteq\langle J\rangle \Leftarrow I_{n-2} \cup\langle I\rangle \subseteq I_{n-2} \cup\langle J\rangle .
$$

It suffices to prove that

$$
I_{n-2} \cup\langle I\rangle \subseteq I_{n-2} \cup\langle J\rangle \Rightarrow I \subseteq J .
$$

Suppose that $I_{n-2} \cup\langle I\rangle \subseteq I_{n-2} \cup\langle J\rangle$. Then $\langle I\rangle \cap J_{n-1}=\left(I_{n-2} \cup\langle I\rangle\right) \cap J_{n-1} \subseteq$ $\left(I_{n-2} \cup\langle J\rangle\right) \cap J_{n-1}=\langle J\rangle \cap J_{n-1}$. Thus, by Lemma 3.3,

$$
I=E\left(\langle I\rangle \cap J_{n-1}\right) \subseteq E\left(\langle J\rangle \cap J_{n-1}\right)=J .
$$

(ii) By (i), we easily deduce that

$$
I=J \Leftrightarrow\langle I\rangle=\langle J\rangle \Leftrightarrow I_{n-2} \cup\langle I\rangle=I_{n-2} \cup\langle J\rangle .
$$

It follows immediately that

$$
I \subset J \Leftrightarrow\langle I\rangle \subset\langle J\rangle \Leftrightarrow I_{n-2} \cup\langle I\rangle \subset I_{n-2} \cup\langle J\rangle .
$$

Now, we can use Lemmas 2.5, 2.8, 3.2 and 3.4 to obtain the following.

Lemma 3.5. For $n \geq 4$ and $s \in[n]$, let $M_{s s}$ be as defined in (2.2). Then $M_{s s}$ is a locally maximal regular subsemiband of $\mathcal{O}_{n}$.

Proof. Recall that $M_{s s}=\left\{\alpha \in \mathcal{O}_{n}: s \alpha=s\right\}$. Let $\alpha \in M_{s s}$. If $|i m(\alpha)|=1$, then clearly $\alpha=\alpha \alpha$ and so $\alpha$ is regular. If $|i m(\alpha)| \geq 2$, suppose that

$$
\alpha=\left(\begin{array}{cccc}
A_{1} & A_{2} & \cdots & A_{r} \\
a_{1} & a_{2} & \cdots & a_{r}
\end{array}\right) \in M_{s s}
$$


where $a_{1}<a_{2}<\cdots<a_{r}, \min A_{i}>\max A_{i-1}, i=2,3, \ldots, r$. Since $\alpha \in M_{s s}$, there exists $k \in\{1,2, \ldots, r\}$ such that $s \in A_{k}$ and $a_{k}=s$. Let

$$
\beta=\left(\begin{array}{llll}
B_{1} & B_{2} & \cdots & B_{r} \\
b_{1} & b_{2} & \cdots & b_{r}
\end{array}\right),
$$

where $b_{k}=s \in A_{k}, b_{i}=\min A_{i}, i \neq k, B_{1}=\left\{1,2, \ldots, a_{1}\right\}, B_{s}=\{x \in[n]$ : $\left.a_{s-1}<x \leq a_{s}\right\}, s=2,3, \ldots, r-1$, and $B_{r}=\left\{a_{r-1}+1, \ldots, n\right\}$, then $\alpha=\alpha \beta \alpha$ and $\beta \in M_{s s}$ (since $s=a_{k} \in B_{k}$ and $b_{k}=s$ ). Then $\alpha$ is regular and by Lemma 2.5 , we have

$$
M_{s s}=\left\langle E\left(J_{n-1}\right) \backslash\{[s \rightarrow s+1],[s \rightarrow s-1]\}\right\rangle .
$$

Thus $M_{s s}$ is a regular subsemiband.

For some $J \subseteq E\left(J_{n-1}\right)$, let $\langle J\rangle$ be a regular subsemiband of $\mathcal{O}_{n}$ properly containing $M_{s s}$, see (3.1). Then, by Lemma 3.4(ii),

$$
E\left(J_{n-1}\right) \backslash\{[s \rightarrow s+1],[s \rightarrow s-1]\} \subset J .
$$

Let $T=I_{n-2} \cup\langle J\rangle$ and let $F_{s}$ be as defined in Lemma 2.8, i.e., $F_{s}=I_{n-2} \cup$ $\left\langle E\left(J_{n-1}\right) \backslash\{[s \rightarrow s+1],[s \rightarrow s-1]\}\right\rangle$, see (3.2). Then, by Lemma 3.4(ii), $F_{s} \subset T$, and since $\langle J\rangle$ is regular, $I_{n-2}$ is a regular semiband (see [2]) and also an ideal of $\mathcal{O}_{n}$, we deduce that $T$ is a regular subsemiband of $\mathcal{O}_{n}$. Thus, by maximality of $F_{s}$ (by Lemma 2.8) and $F_{s} \subset T, T=I_{n-2} \cup\langle J\rangle=\mathcal{O}_{n}$. It now follows immediately that $E\left(J_{n-1}\right) \subseteq\langle J\rangle$ and so $\left\langle E\left(J_{n-1}\right)\right\rangle \subseteq\langle J\rangle$. Thus, by Lemma $3.2,\langle J\rangle=\mathcal{O}_{n}$.

Also, using Lemmas 2.5, 2.8, 3.2 and 3.4, we have:

Lemma 3.6. For $n \geq 4$ and $2 \leq s \leq n-2$, let $M_{s(s+1)}$ be as defined in (2.2). Then $M_{s(s+1)}$ is a locally maximal regular subsemiband of $\mathcal{O}_{n}$.

Proof. Recall that $M_{s(s+1)}=\left\{\alpha \in \mathcal{O}_{n}: s \alpha \leq s,(s+1) \alpha \geq s+1\right\}$. Note that for any $\alpha \in M_{s(s+1)},|i m(\alpha)| \geq 2$ (since $s \alpha \leq s$ and $\left.(s+1) \alpha \geq s+1\right)$. Consider a typical element

$$
\alpha=\left(\begin{array}{llll}
A_{1} & A_{2} & \cdots & A_{r} \\
a_{1} & a_{2} & \cdots & a_{r}
\end{array}\right) \in M_{s(s+1)},
$$

where $a_{1}<a_{2}<\cdots<a_{r}, \min A_{i}>\max A_{i-1}, i=2, \ldots, r$. Since $\alpha \in M_{s(s+1)}$, there exist $k \in\{1,2, \ldots, r-1\}$ such that $s \in A_{k}, s+1 \in A_{k+1}$ and $a_{k} \leq s<$ $s+1 \leq a_{k+1}$. Let $c_{i}=a_{i}(i \neq k)$ and $c_{k}=s$, then $c_{1}<c_{2}<\cdots<c_{r}$. Let

$$
\beta=\left(\begin{array}{llll}
B_{1} & B_{2} & \cdots & B_{r} \\
b_{1} & b_{2} & \cdots & b_{r}
\end{array}\right),
$$

where $b_{k+1}=s+1 \in A_{k+1}, b_{i}=\min A_{i}, i \neq k+1, B_{1}=\left\{1,2, \ldots, c_{1}\right\}$, $B_{i}=\left\{x \in[n]: c_{i-1}<x \leq c_{i}\right\}, i=2,3, \ldots, r-1$ and $B_{r}=\left\{c_{r-1}+1, \ldots, n\right\}$. Clearly, $\beta \in \mathcal{O}_{n}$. Note that $s=c_{k} \in B_{k}$ and $s+1 \in B_{k+1}$ (since $c_{k}=s<$ $\left.s+1 \leq a_{k+1}=c_{k+1}\right)$. It follows that $s \beta=B_{k} \beta=b_{k}=\min A_{k} \leq s$ (since $\left.s \in A_{k}\right)$ and $(s+1) \beta=B_{k+1} \beta=b_{k+1}=s+1$. Thus $\beta \in M_{s(s+1)}$. Note that $c_{i}=a_{i}(i \neq k)$ and $a_{k} \leq s=c_{k}<s+1 \leq a_{k+1}$. It follows that $a_{i} \in B_{i}$ 
$(i=1,2, \ldots, r)$ and so $\alpha=\alpha \beta \alpha$. Thus $\alpha$ is regular and by Lemma 2.5, we have

$$
M_{s(s+1)}=\left\langle E\left(J_{n-1}\right) \backslash\{[s \rightarrow s+1],[s+1 \rightarrow s]\}\right\rangle .
$$

Thus $M_{s(s+1)}$ is a regular subsemiband.

For some $J \subseteq E\left(J_{n-1}\right)$, let $\langle J\rangle$ be a regular subsemiband of $\mathcal{O}_{n}$ properly containing $M_{s(s+1)}$, see (3.3). Then, by Lemma 3.4(ii),

$$
E\left(J_{n-1}\right) \backslash\{[s \rightarrow s+1],[s+1 \rightarrow s]\} \subset J .
$$

Let $T=I_{n-2} \cup\langle J\rangle$ and let $G_{s}$ be as defined in Lemma 2.8, i.e., $G_{s}=I_{n-2} \cup$ $\left\langle E\left(J_{n-1}\right) \backslash\{[s \rightarrow s+1],[s+1 \rightarrow s]\}\right\rangle$. Then, by Lemma 3.4(ii), $G_{s} \subset T$. Since $\langle J\rangle$ is regular, $I_{n-2}$ is a regular semiband (see [2]) and also an ideal of $\mathcal{O}_{n}$, we deduce that $T$ is a regular subsemiband of $\mathcal{O}_{n}$. Thus, by maximality of $G_{s}$ (by Lemma 2.8) and $G_{s} \subset T, T=I_{n-2} \cup\langle J\rangle=\mathcal{O}_{n}$. It follows immediately that $E\left(J_{n-1}\right) \subseteq\langle J\rangle$ and so $\left\langle E\left(J_{n-1}\right)\right\rangle \subseteq\langle J\rangle$. Thus, by Lemma $3.2,\langle J\rangle=\mathcal{O}_{n}$.

The following lemma gives a necessary condition for a locally regular subsemiband of $\mathcal{O}_{n}$ to be maximal.

Lemma 3.7. Let $I$ be a nonempty set of $E\left(J_{n-1}\right)$. If $\langle I\rangle$ is a locally maximal regular subsemiband of $\mathcal{O}_{n}$, then $T=I_{n-2} \cup\langle I\rangle$ is a maximal regular subsemiband of $\mathcal{O}_{n}$.

Proof. Suppose that $\langle I\rangle$ is a locally maximal regular subsemiband of $\mathcal{O}_{n}$. Let $M$ be a regular subsemiband of $\mathcal{O}_{n}$ properly containing $T$. Since $M=\langle E(M)\rangle$ and $I_{n-2} \subseteq M($ since $T \subset M)$, we have $M=I_{n-2} \cup M=I_{n-2} \cup\left\langle E\left(M \cap J_{n-1}\right)\right\rangle$ and so

$$
I_{n-2} \cup\langle I\rangle=T \subset M=I_{n-2} \cup\left\langle E\left(M \cap J_{n-1}\right)\right\rangle .
$$

Note that $E\left(M \cap J_{n-1}\right) \subseteq E\left(J_{n-1}\right)$. Then, by Lemma 3.4(ii), $\langle I\rangle \subset\langle E(M \cap$ $\left.\left.J_{n-1}\right)\right\rangle$ and so, by the locally maximality of $\langle I\rangle,\left\langle E\left(M \cap J_{n-1}\right)\right\rangle=\mathcal{O}_{n}$. Thus $M=\mathcal{O}_{n}$ and so $T=I_{n-2} \cup\langle I\rangle$ is a maximal regular subsemiband of $\mathcal{O}_{n}$.

Now, we can prove Theorem 3.1.

Proof Theorem 3.1. Let $M_{s s}$ and $M_{s(s+1)}$ be defined earlier. It is obvious that

$$
\begin{gathered}
A_{s}=\left\{\alpha \in \mathcal{O}_{n}: s \alpha=s\right\}=M_{s s}, \\
B_{s}=\left\{\alpha \in \mathcal{O}_{n}: s \alpha \leq s,(s+1) \alpha \geq s+1\right\}=M_{s(s+1)} .
\end{gathered}
$$

Thus, by Lemmas 3.5 and 3.6, $A_{s}$ and $B_{s}$ are locally maximal regular subsemibands of $\mathcal{O}_{n}$.

Conversely, we shall prove that each locally maximal regular subsemiband of $\mathcal{O}_{n}$ must be of the form $A_{s}$ or $B_{s}$. For some $I \subseteq E\left(J_{n-1}\right)$, let $\langle I\rangle$ is a locally maximal regular subsemiband of $\mathcal{O}_{n}$. Then, by Lemma 3.7, $T=I_{n-2} \cup\langle I\rangle$ is a maximal regular subsemiband of $\mathcal{O}_{n}$. Thus, by Lemma 2.8, there exists $s \in[n]$ such that $T=F_{s}=I_{n-2} \cup\left\langle E\left(J_{n-1}\right) \backslash\{[s \rightarrow s+1],[s \rightarrow s-1]\}\right\rangle$ or there exists 
$s \in\{2,3, \ldots, n-2\}$ such that $T=G_{s}=I_{n-2} \cup\left\langle E\left(J_{n-1}\right) \backslash\{[s \rightarrow s+1],[s+1 \rightarrow\right.$ $s]\}\rangle$. It follows immediately from Lemmas 3.4 that

$$
\begin{aligned}
& \langle I\rangle=\left\langle E\left(J_{n-1}\right\rangle \backslash\{[s \rightarrow s+1],[s \rightarrow s-1]\}\right\rangle \text { or } \\
& \langle I\rangle=\left\langle E\left(J_{n-1}\right) \backslash\{[s \rightarrow s+1],[s+1 \rightarrow s]\}\right\rangle .
\end{aligned}
$$

Thus, by Lemma 2.5 and (3.4), (3.5),

$$
\begin{aligned}
& \langle I\rangle=\left\langle E\left(J_{n-1}\right\rangle \backslash\{[s \rightarrow s+1],[s \rightarrow s-1]\}\right\rangle=M_{s s}=A_{s} \text { or } \\
& \langle I\rangle=\left\langle E\left(J_{n-1}\right) \backslash\{[s \rightarrow s+1],[s+1 \rightarrow s]\}\right\rangle=M_{s(s+1)}=B_{s} .
\end{aligned}
$$

Acknowledgments. The authors wish to express their appreciation to the referees for some valuable comments and suggestions that helped to improve the presentation of this paper.

\section{References}

[1] A. Ya. Aizenstat, The defining relations of the endomorphism semigroup of a finite linearly ordered set, Sibirsk. Mat. 3 (1962), 161-169.

[2] G. U. Garba, On the idempotent ranks of certain semigroups of order-preserving transformations, Portugal. Math. 51 (1994), no. 2, 185-204.

[3] G. M. S. Gomes and J. M. Howie, On the ranks of certain semigroups of order-preserving transformations, Semigroup Forum 45 (1992), no. 3, 272-282.

[4] P. M. Higgins, Idempotent depths in semigroups of order-preserving mappings, Proc. Roy. Soc. Edinburgh Sect. A 124 (1994), no. 5, 1045-1058.

[5] J. M. Howie, Products of idempotents in certain semigroups of transformation, Proc. Edinburgh Math. Soc. 17 (1971), 223-236.

[6] A. Laradji and A. Umar, Combinatorial results for semigroups of order-preserving full transformations, Semigroup Forum 62 (2006), no. 1, 51-62.

[7] F. Pastijin, Embedding semigroups in semibands, Semigroup Forum 14 (1977), no. 3, 247-263.

[8] A. Umar, On the semigroups of order-decreasing finite full transformations, Proc. Roy. Soc. Edinburgh Sect. A 120 (1992), no. 1-2, 129-142.

[9] B. Xu, P. Zhao, and J. Y. Li, Locally maximal idempotent-generated subsemigroups of singular order-preserving transformation semigroups, Semigroup Forum 72 (2006), no. 3, 488-492.

[10] X. L. Yang, On the nilpotent ranks of the principal factors of order-preserving transformation semigroups, Semigroup Forum 57 (1998), no. 3, 331-340.

[11] - Products of idempotents of defect 1 in certain semigroups of transformations, Comm. Algebra 27 (1999), no. 7, 3557-3568.

[12] X. L. Yang and C. H. Lu, Maximal properties of some subsemigroups in finite orderpreserving transformation semigroups, Comm. Algebra 28 (2000), no. 7, 3125-3135.

[13] P. Zhao, B. Xu, and M. Yang, A note on maximal properties of some subsemigroups of order-preserving transformation semigroups, Comm. Algebra 40 (2012), no. 3, 11161121. 
Ping ZHAO

School of Mathematics and Computer Science

GuiZhou Normal University

Guiyang, GuiZhou Province 550001, P. R. China

AND

Mathematics Teaching \& Research Section

Guiyang Medical College

Guiyang, GuiZhou Province 550004, P. R. China

E-mail address: zhaoping731108@hotmail.com, peaceguorong@yahoo.com

MeI YANG

Cadre Proppants, Houston, Texas, 77079, USA

E-mail address: mei.yang@cadreproppants.com 